\title{
An N-band test bench for the METIS coronagraphic masks
}

Ronayette, Samuel, Mouzali, Salima, Barrière, JeanChristophe, Pantin, Éric, Orduna, Thierry, et al.

Samuel Ronayette, Salima Mouzali, Jean-Christophe Barrière, Éric Pantin, Thierry Orduna, Pascal Gallais, Michel Lortholary, Luc Dumaye, Olivier Absil, Christian Delacroix, Derek Ives, Mikael Karlsson, "An N-band test bench for the METIS coronagraphic masks," Proc. SPIE 11447, Ground-based and Airborne Instrumentation for Astronomy VIII, 114472 U (15 December 2020); doi: $10.1117 / 12.2562261$ 


\title{
An N-band test bench for the METIS coronagraphic masks
}

\author{
Samuel Ronayette ${ }^{\mathrm{a}}$, Salima Mouzali ${ }^{\mathrm{a}}$, Jean-Christophe Barrière ${ }^{\mathrm{a}}$, Éric Pantin ${ }^{\mathrm{a}}$, Thierry \\ Orduna $^{\mathrm{a}}$, Pascal Gallais ${ }^{\mathrm{a}}$, Michel Lortholary ${ }^{\mathrm{a}}$, Luc Dumaye ${ }^{\mathrm{a}}$, Olivier Absil ${ }^{\mathrm{b}}$, Christian \\ Delacroix $^{\mathrm{b}}$, Derek Ives ${ }^{\mathrm{c}}$, and Mikael Karlsson ${ }^{\mathrm{d}}$ \\ ${ }^{a}$ CEA Saclay, DRF/IRFU/DAp, 91191 Gif-sur-Yvette Cedex, France \\ bSTAR Institute, University of Liège, 19C allée du Six Août, B-4000 Liège, Belgium \\ ${ }^{c}$ European Southern Observatory, Karl-Schwarzschild-Str. 2, 85748 Garching, Germany \\ ${ }^{\mathrm{d}}$ Department of Materials Science and Engineering, Uppsala University, P.O. Box 35, SE-751 \\ 03 Uppsala, Sweden
}

\begin{abstract}
METIS is one of the first three instruments for the ELT, Europe's next-generation ground-based telescope. It will offer imaging, coronagraphy and spectroscopy in the L, M and $\mathrm{N}$ bands for general-purpose science in astrophysics. Among its main science drivers are circumstellar disks and extrasolar planets observations, which requires demanding high contrast imaging techniques. In that framework, METIS will be equipped with state-of-the-art phase mask coronagraphs: Apodizing Phase Plate (APP) and Annular Grooves Phase Mask (AGPM). Manufacturing the AGPM coronagraphs is a complex process that requires performance assessment with specific testing before implementation into the instrument. At Department of Astrophysics (CEA Saclay, France), responsible for the testing of the N-band AGPMs, a previously available test bench with a telescope simulator and cryogenic facility has been upgraded to comply with the AGPM tests requirements. This paper presents these requirements and describes the test bench design adopted. Then, based on preliminary results, we discuss the original solutions that permitted to reach our goals.
\end{abstract}

Keywords: Coronagraphy, Annular Groove Phase Mask, METIS, E-ELT, infrared, test bench, QCL Lasers, N-Band

\section{INTRODUCTION}

Testing coronagraphs is a demanding work on many aspects. It requires point-like source imaging with high optical quality, a very fine positioning accuracy and a high signal-to-noise ratio (SNR). The whole system must be very stable over several minutes, or even hours, of time, and the measurements repeatable. The high extinction level $\left(>10^{3}\right)$ we want to measure requires a large dynamic range. Furthermore, in our case, working in the midinfrared spectral band adds technical difficulties, with the need of a cooled down instrument, adapted light sources, and a system for background subtraction at a sufficiently high frequency $(\approx 0.2-2 \mathrm{~Hz})$.

A test bench used for the verification of the JWST/MIRI imager (MIRIM) had been developed at Department of Astrophysics of CEA Saclay. This test bench was compliant with the requirements for testing the coronagraphic mode of MIRI, in the mid-infrared range. It therefore appeared natural to use it for the testing of the METIS coronagraph, but it first needed an upgrade. The detector had to be changed, the sources adapted to the high SNR required, the cryostat refurbished with cryocoolers, in replacement of the liquid helium vessel.

The test bench now hosts the METIS-PDR baseline detector for the N-band, an Aquarius mid-infrared array (Raytheon Si:As 1kx1k BIB). Laser sources come in replacement of the traditional blackbody. Indeed, mid-infrared QCL lasers offer an affordable solution with a variety of wavelengths. Together with the large capacitance detector, they provide high fluxes to reach the requested signal-to-noise ratio. Coupled to singlemode optical fibers, which are now commonly available for wavelengths up to $16 \mu \mathrm{m}$ (hollow-core waveguides), these lasers can be of great interest for the coronagraphs characterization.

Further author information: (Send correspondence to S. Ronayette)

S. Ronayette: E-mail: samuel.ronayette@cea.fr, Telephone: +33 (0) 169084282

Ground-based and Airborne Instrumentation for Astronomy VIII, edited by Christopher J. Evans,

Julia J. Bryant, Kentaro Motohara, Proc. of SPIE Vol. 11447, 114472U · C 2020

SPIE · CCC code: $0277-786 \mathrm{X} / 20 / \$ 21 \cdot$ doi: $10.1117 / 12.2562261$

Proc. of SPIE Vol. 11447 114472U-1 


\section{TEST BENCH DESCRIPTION}

\subsection{Requirements}

To make the performance assessment of the coronagraphic masks possible, the test bench must comply with a set of optical, thermal and mechanical requirements. The main requirements are given below.

1. Spectral band: covers the range from 8 to $13 \mu \mathrm{m}$.

2. Maximum wavefront error: $200 \mathrm{~nm}$ RMS.

3. PSF: oversampled, at least $2 \mathrm{x}$ Nyquist at $\lambda=10 \mu \mathrm{m}$.

4. Focusing capabilities: at least $300 \mu \mathrm{m}$ range, $100 \mu \mathrm{m}$ accuracy.

5. Point source movement: positioning accuracy of about $0.01 \lambda / D$.

6. Filters: the AGPM must be tested in 5 spectral bands with a spectral resolution $\mathrm{R}=10$ at least.

7. A cold pupil plane must be fitted with Lyot masks of diameter undersized by $10 \%$ compared to the pupil image.

8. The source power, optics throughput and detection chain sensitivity, must be such that a SNR of 10,000 can be reached in 1 minute. This will enable to measure a $10^{-3}$ peak null depth with an SNR of 3 on the timescale of a few seconds, and thereby enable an efficient centering process.

9. The point source shall be unresolved compared to the instrument diffraction, i.e. the diameter of the source shall be smaller than $0.1 \lambda / D$.

\subsection{Overview of the set-up}

A functional diagram of the test bench in shown in Fig. 1. The test bench includes the following items:

- A point source, made by a pinhole illuminated by an infrared source (laser via optical fiber)

- A hexapod, which allows movements across the instrument field of view and focus adjustment of the point source.

- A flat source (selected instead of the point source), which illuminates the whole field of view with a blackbody-like radiation (up to $90 \mathrm{~K}$ above ambient temperature)

- The telescope simulator optics, which make an image of the pinhole in the cryostat, at the instrument focal plane.

- A mask in the optical path, which sets the pupil aperture shape and position.

- Finally, the MIRI ETM (Engineering and Test Model) which forms an image of the focal plane on the Aquarius detector. The instrument is equipped with a filter wheel.

To dim the air turbulence and improve stability of the point source, the whole bench is covered with a plastic sheet.

Several distinct computers provide control command of the test bench.

A first computer, the OGSE* computer, controls everything directly related to the telescope simulator, plus the filter wheel in the cryostat. The following features are directly controlled by this computer:

- Selection of point source or flat source

- Hexapod (point source) position with 6 degrees of freedom

- Extended source temperature

- Warm pupil position

- Cold filter wheel position

A second computer, the NGC (see Sec. 2.6), hosts the detector control system (DCS), which permits the image acquisition, by controlling and reading out the detector. A frequency generator can be used to trigger data acquisition synchronously with a chopper in the telescope simulator. The chopper is placed upstream of the pinhole and cannot be seen by the instrument. Therefore, the source can be alternatively turned on and off while leaving the background unchanged, allowing efficient background subtraction.

\footnotetext{
*OGSE stands for Optical Ground Support Equipment. This naming inherits the JWST/MIRI days, to distinguish the ground test equipment from the space instrument
} 


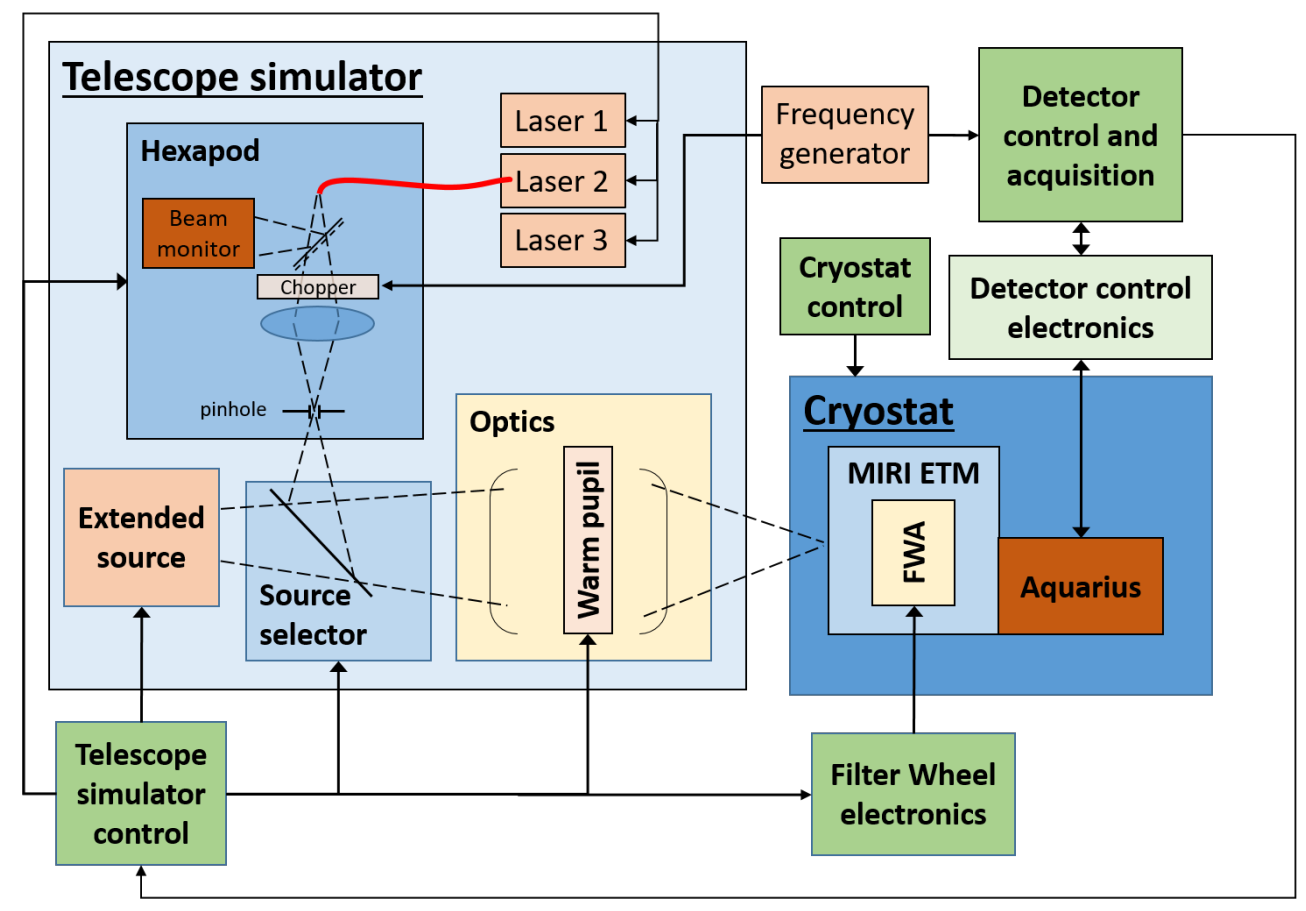

Figure 1. Synoptic of the IR test bench facility. The green boxes identify control electronics or computers. The orange boxes are devices driven by these controller. The arrows indicate an existing communication protoctol, either uni- or bidirectional.

Furthermore, the DCS is interfaced with the OGSE computer. Complex acquisition scripts can be written to perform, for instance, scanning of the source in the entire field of view, in an automated way.

Finally, an automatic system controls the cryostat temperature.

\subsection{The telescope simulator}

\subsubsection{Sources}

The use of a classical source has been studied. The test bench is equipped with an IR emitter, a heated ceramic that is refocused on a pinhole. It appears that this solution faces two issues:

1. To meet the requirement on the maximum point source diameter (see Sec. 2.1, point 9), a very small pinhole (typically $10 \mu \mathrm{m}$ ) must be used. In consequence, the flux passing through becomes very small and the requirement on the SNR cannot be met.

2. The source being broad band, the out of band rejection of the filters must be very high, on a large spectral band, in order to be able to measure nulling to the level of $10^{-3}$. This meant for us to buy an entire new set of custom made filters which are very costly.

Consequently, we decided to use laser sources. They are intrinsically narrow-band, and can be used with standard, large band filters. They are powerful, and the requirement on the SNR is easily met. We chose to use a set of Quantum Cascade Lasers (QCL), one for each wavelength to be tested, coupled to a single mode fiber. The lasers are made by Alpes Laser, and the fiber comes from Opto-knowledge. A QCL in its housing is shown in Fig. 2. To improve stability of the signal, we mounted both the lasers and the fiber on the hexapod table, so the fiber does not move at all when the hexapod moves. We indeed noticed that changing the bending of the fiber leads to important flux variation at the fiber output, which we want the avoid. This is shown in Fig. 3. 

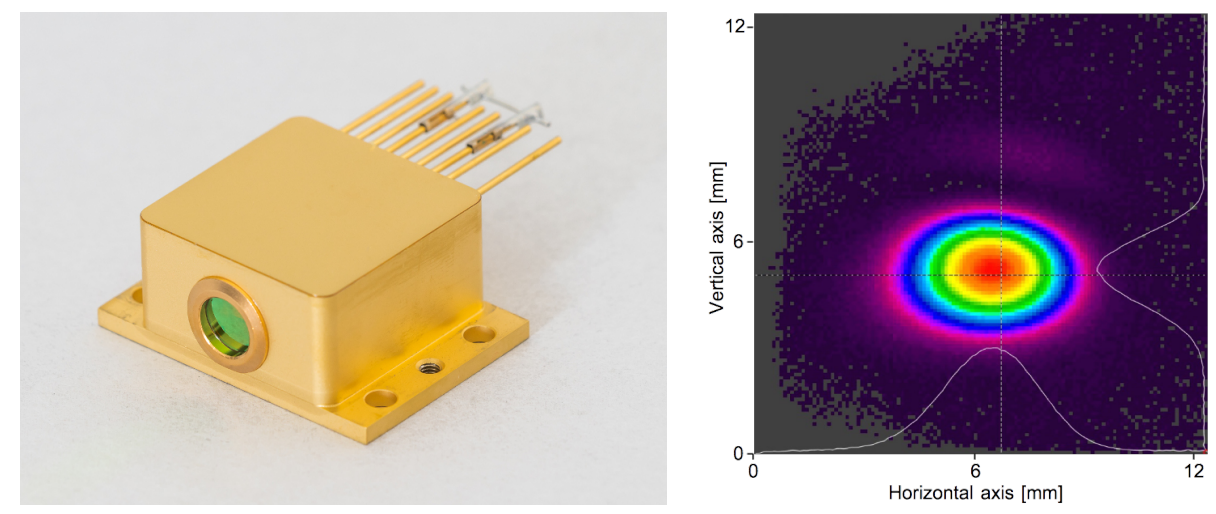

Figure 2. Left. A QCL laser in its housing. One can see the integrated lens used for a collimating the output beam. Right. Typical beam profile of a collimated QCL. Photos courtesy of Alpes Lasers.

\subsubsection{Hexapod}

The hexapod is an accurate positioning system that holds the "source assembly", i.e. the infrared emitter, the optics relay, the shutter and the pinhole. It has six degrees of freedom, to place the source anywhere in the instrument field of view ( $\mathrm{x}, \mathrm{y})$, with focus adjustment $(\mathrm{z})$. Furthermore, tip and tilt movements $\left(\theta_{x}, \theta_{y}\right)$ set the direction of the optical axis, so it is always pointing towards the center of the entrance pupil, the optical system being not telecentric. This ensure that the illumination pattern is well centered on the pupil. The sixth degree of freedom $\left(\theta_{z}\right.$, rotation around the optical axis) is not used.

Table 1 gives the characteristics of the movements along each degree of freedom. Various accuracies have been specified to the manufacturer, within various ranges. It comes from the need to have a very good accuracy in a small range near the center of the coronagraphic masks. However, across the whole field of view, such an accuracy is not necessary and the requirement can be relaxed.

\subsubsection{Optics}

The optical layout of the telescope simulator is shown in Fig. 4. A first off-axis parabola (OAP1) collimates the beam from the point source. The pupil mask is placed in the collimated beam. A second off-axis parabola

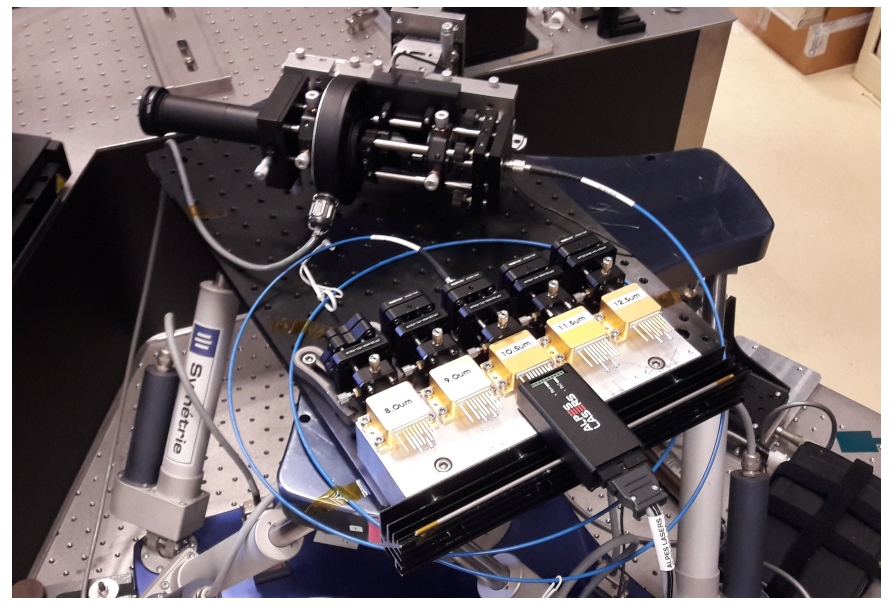

Figure 3. The QCLs mounted on the hexapod. One can see the Alps Laser pulser, which powers the laser, followed by the laser itself. Then the optical fibre carries the signal to the optical relay, which focuses the beam on the pinhole, that is the entry port of the telescope simulator. For chopping, a shutter is placed in the collimated part of the optical relay. 
Table 1. Characteristics of the hexapod movements

\begin{tabular}{|c|c|c|c|c|}
\hline Movement & Combined range* & Accuracy & Repeatability & Stability \\
\hline$T_{x}$ and $T_{y}$ & $\pm 41 \mathrm{~mm}$ & $\begin{array}{c} \pm 500 \mu \mathrm{m} \\
( \pm 3 \mu \mathrm{m} \text { within } \\
\pm 150 \mu \mathrm{m} \text { range })\end{array}$ & $\pm 1 \mu \mathrm{m}$ & $\begin{array}{c} \pm 3 \mu \mathrm{m} \\
\text { during } 15 \mathrm{~min}\end{array}$ \\
\hline$T_{z}$ (focus) & $\pm 7 \mathrm{~mm}$ & $\pm 50 \mu \mathrm{m}$ & $\pm 1 \mu \mathrm{m}$ & $\begin{array}{c} \pm 100 \mu \mathrm{m} \\
\text { during } 15 \mathrm{~min}\end{array}$ \\
\hline$\theta_{x}$ and $\theta_{y}$ & $\pm 1^{\circ}$ & \pm 6 & $\pm 10^{\prime \prime}$ & - \\
\hline
\end{tabular}

*This is the larger volume combining all the degrees of freedom at their maximum extent. Each movement individually has a larger range when other movements remain at their central position

(OAP2) focuses the beam inside the cryostat, at the entrance focal plane of the instrument. Two flat mirrors fold the beam.

The aperture size of the pupil mask sets the F-number of the beam. The distance between the pupil mask and OAP2 is set so the exit pupil is $3040 \mathrm{~mm}$ before the focal surface, which corresponds the entrance pupil position of the MIRIM instrument.

The nominal aperture of the beam is $\mathrm{F} / 20$, but larger pupil mask can be used to have beams up to $\mathrm{F} / 15$ with no vignetting, or even $\mathrm{F} / 12$ if one is interested only in the central part of the field of view (vignetting appears rapidly in the field with this aperture). Table 2 gives the pupil mask diameter and PSF size for different beam apertures that will be used during the tests. The F/30 beam leads to a comfortable sampling of the PSF, while the F/20 beam gives a PSF size closer to the "real" PSF for METIS.

The system is diffraction limited, with spot size between 3 and $13 \mu \mathrm{m}$ RMS across the whole field of view $\left(72 \times 72 \mathrm{~mm}^{2}\right)$, with an F/20 beam. See Fig. 4 .

Table 2. Pupil mask diameter and PSF size for different beam apertures in the telescope simulator. The real size of the PSF and its size in terms of Nyquist sampling are given (pixels of $84 \mu \mathrm{m}$, corresponding to the size of the pixels of the Aquarius detector as imaged by the MIRIM optics)

\begin{tabular}{|c|c|c|}
\hline Beam aperture & Pupil mask diameter $\mathbf{( m m})$ & PSF FWHM at $\mathbf{1 0} \mu \mathbf{m}$ \\
\hline $\mathrm{F} / 20$ & 75.4 & $200 \mu \mathrm{m}(1.2 \mathrm{x}$ Nyquist $)$ \\
\hline $\mathrm{F} / 30$ & 50.3 & $300 \mu \mathrm{m}(1.8 \mathrm{x}$ Nyquist $)$ \\
\hline
\end{tabular}

\subsection{The cryogenic facility}

The cryogenic facility is composed of:

- A vaccuum vessel

- coolers

- automated controller

The vaccuum vessel hosts the MIRIM imager (see Sec. 2.5). MIRIM is mounted on a plate that is cooled downed to $5-10 \mathrm{~K}$, and is shrouded by two screens at $4 \mathrm{~K}$ and $40 \mathrm{~K}$. A neutral density is mounted on the $4 \mathrm{~K}$ screen, to diminish the thermal background from the outside world, and avoid detector saturation. The optical port of the cryostat is a germanium window, $12 \mathrm{~mm}$ thick and $170 \mathrm{~mm}$ large. A knife gate valve is used to change the window without warming up the cryostat. It allows us to have specific windows for each wavelength range of interest. Finally, the vessel in mounted on a manual hexapod for fine positioning, which is required for the alignment with the telescope simulator. 

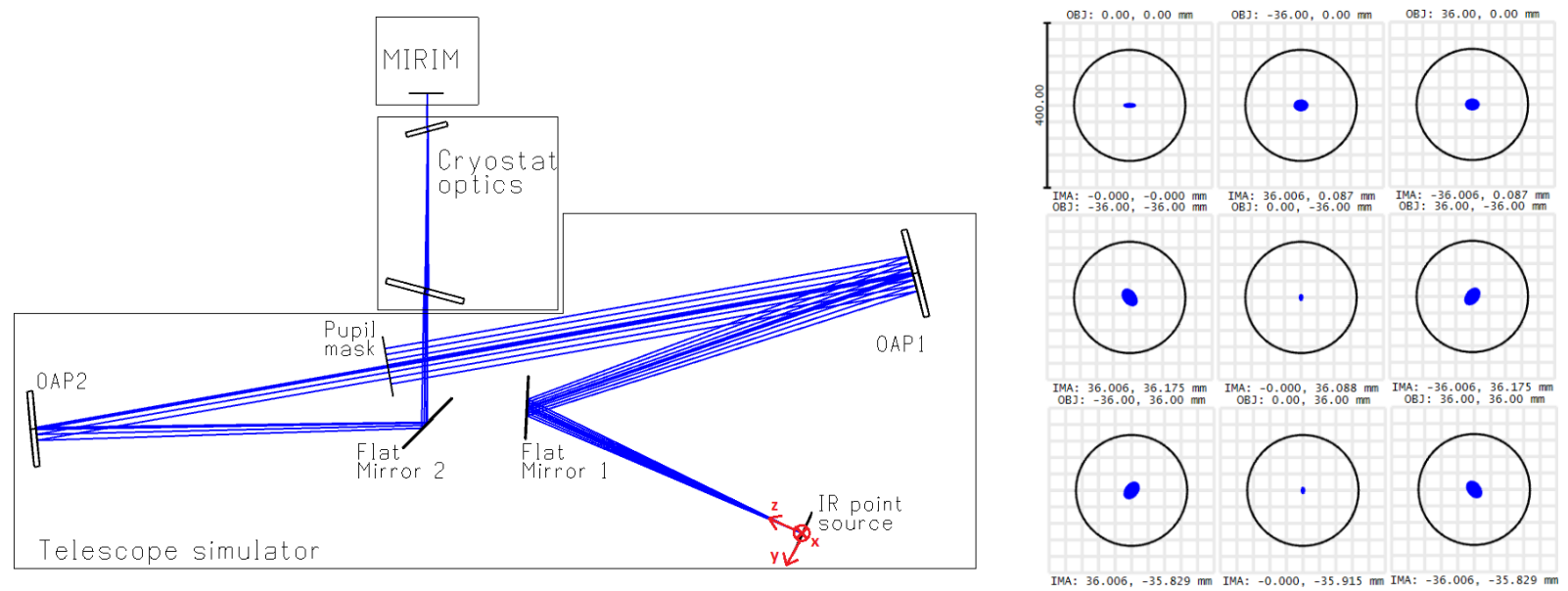

Figure 4. Left. Optical layout of the telescope simulator, from the source down to the entrance focal plane of the MIRI imager. Right. Spot diagram of the telescope simulator with an F/20 beam. The circles give the Airy pattern diameter, which is $270 \mu \mathrm{m}$ at $\lambda=5.6 \mu \mathrm{m}$. All spots are smaller than $13 \mu \mathrm{m} \mathrm{rms}$. The optical system is diffraction limited.

Originally, the cryostat was cooled down with liquid helium. In the scope of the upgrade for the METIS project, this system has been replaced with pulse tube cryocoolers. If the vibration generated by the pulse tube appears to be disturbing for our measurements, they can be switched of for about 20 minutes.

The programmable controller automatically supervises the cryostat. It reads the temperatures, regulates the heaters, controls the gates. Complex phases, like cooling down and warming up, that requires to follow a sequence of operations, are performed by the controller.

With this upgrade, the cryostat facility became an autonomous system capable of running $24 \mathrm{~h}$ a day, 7 days a week without any human intervention. Figure 5 shows the cryostat vacuum vessel and the electronic bay hosting the controller.

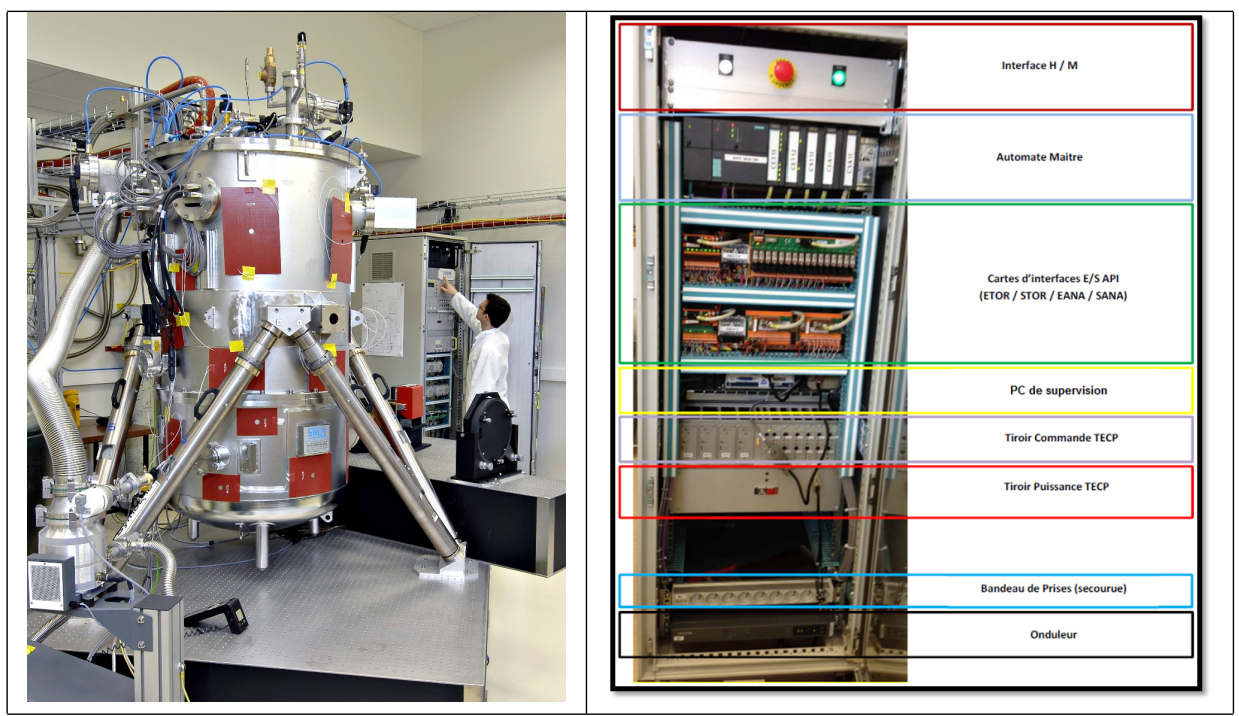

Figure 5. Left. The cryostat vessel. Right. The electronic bay of the cryostat controller. 


\subsection{The infrared imager}

\subsubsection{Optics}

MIRIM, the MIRI imager, is an infrared instrument working in the spectral range $5-30 \mu m$. Originally, it features three observing modes (imaging, coronagraphy, low-resolution spectroscopy), plus an alignment check mode and an optical path for a calibration beam. In the scope of the METIS N-band test bench, only the coronagraphic mode is relevant.

The optical design is an all-reflective design including a collimator, a folding mirror, and a Three Mirrors Anastigmatic (TMA) objective. The collimating mirror provides a pupil image of good quality to locate a fixed cold stop (19 $\mathrm{mm}$ in diameter). The TMA provides a very good image quality at the image plane. See Fig. 6 .

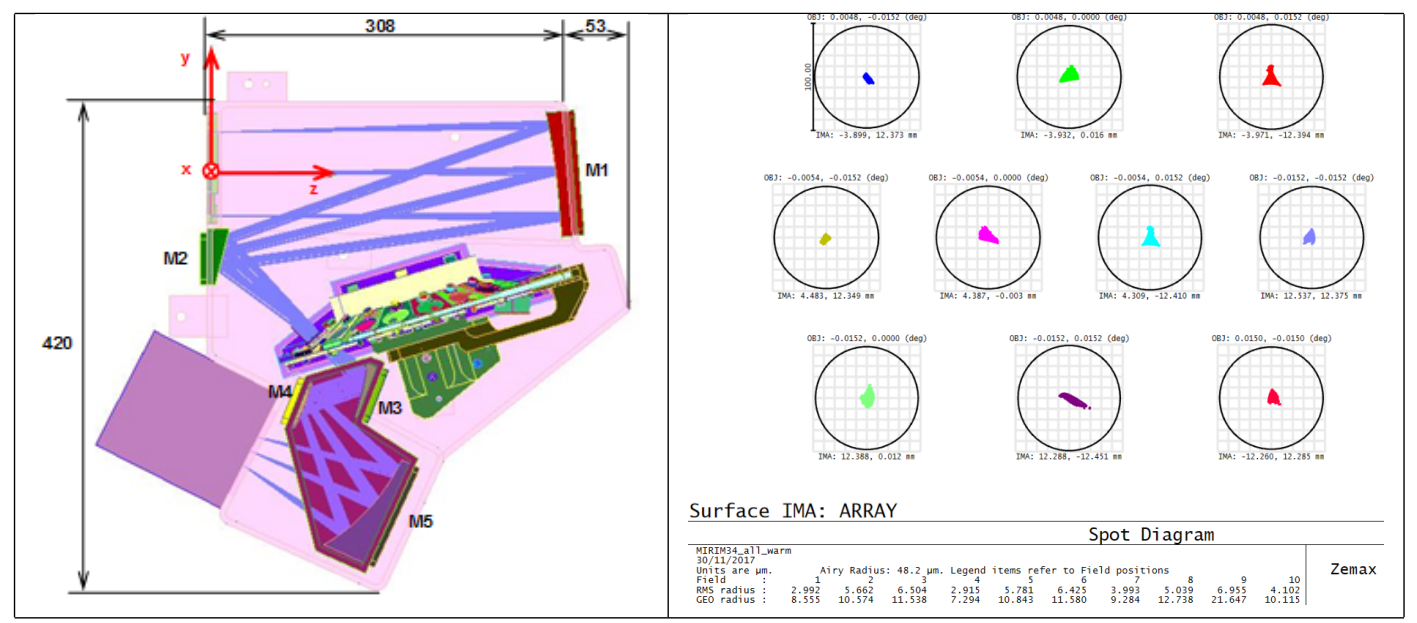

Figure 6. Left. Mechanical and optical layout of the MIRIM Imager. Right. Spot diagram of the MIRI Imager. The quality is diffraction limited in the entire field of view.

The filter wheel has 18 positions, for the imaging mode (10 filters), the coronagraphy (4 filters) and the low-resolution spectroscopy (1 prism). The remaining positions host a neutral density, the alignment lens and a closed position. See also section 2.5.3.

Table 3 gives the main optical characteristics of the instrument.

Table 3. Main optical characteristics of the MIRI imager

\begin{tabular}{|c|c|c|c|}
\hline Field of view & Magnification & working F\# & Spatial resolution \\
\hline $71.6 \times 71.6 \mathrm{~mm}^{2}$ & -0.357 & 7.05 & $\begin{array}{c}F W H M \approx \lambda F=70 \mu \mathrm{m} \text { at } 10 \mu \mathrm{m} \\
\text { (Nyquist at } \lambda=8.5 \mu \mathrm{m} \text { with } 30 \mu \mathrm{m} \text { pixels). }\end{array}$ \\
\hline
\end{tabular}

\subsubsection{Focal plane}

The focal plane of the instrument is equipped with the AGPM masks. For that purpose, the focal plane assembly of MIRI that holds the coronagraphs masks is removed, and replaced by a newly designed one. Figure 7 shows the position of the masks in this new mount.

\subsubsection{Filters}

The filter wheel is equipped with the filters and diaphragm as shown in Fig. 8. Table 4 lists the filters that will be used for the METIS testing in the N-band. Because of the use of laser sources, narrow filters with high out-of-band rejection are no longer needed. Passband filters such as the MIRIM imaging filters, are suitable. However, two coronagraphic filters (at 10.65 and $11.4 \mu \mathrm{m}$ ) are available, and equipped with a circular diaphragm. They have 


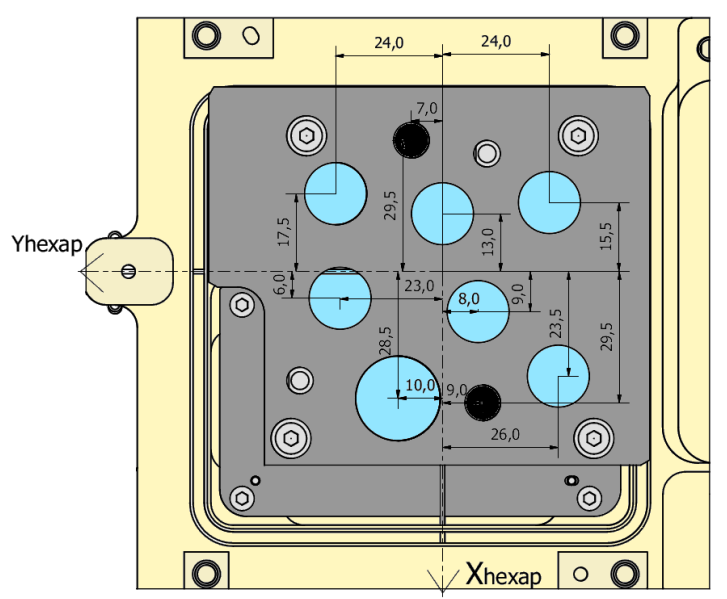

Figure 7. The entrance focal plane of MIRIM, with the new mount for the AGPMs. Up to 6 masks of diameter 15 mm +1 mask of diameter $20 \mathrm{~mm}$ can be mounted. The mask positions have been chosen such that the central line of the detector is avoided, and their centers lie on different pixels columns.

a high out-of-band rejection, and may be used for coronagraphic measurements with a standard blackbody-like source.

\subsubsection{Coronagraphic diaphragms}

New coronagraphic diaphragms will be manufactured and mounted, adapted to the new pupil shape. The pupil is a plain circle. For good coronagraphic performances, the diaphragms have to be undersized to $90 \%$ of the pupil diameter. Because we plan to perform tests with different pupil apertures (see Sec. 2.3.3), the wheel will be

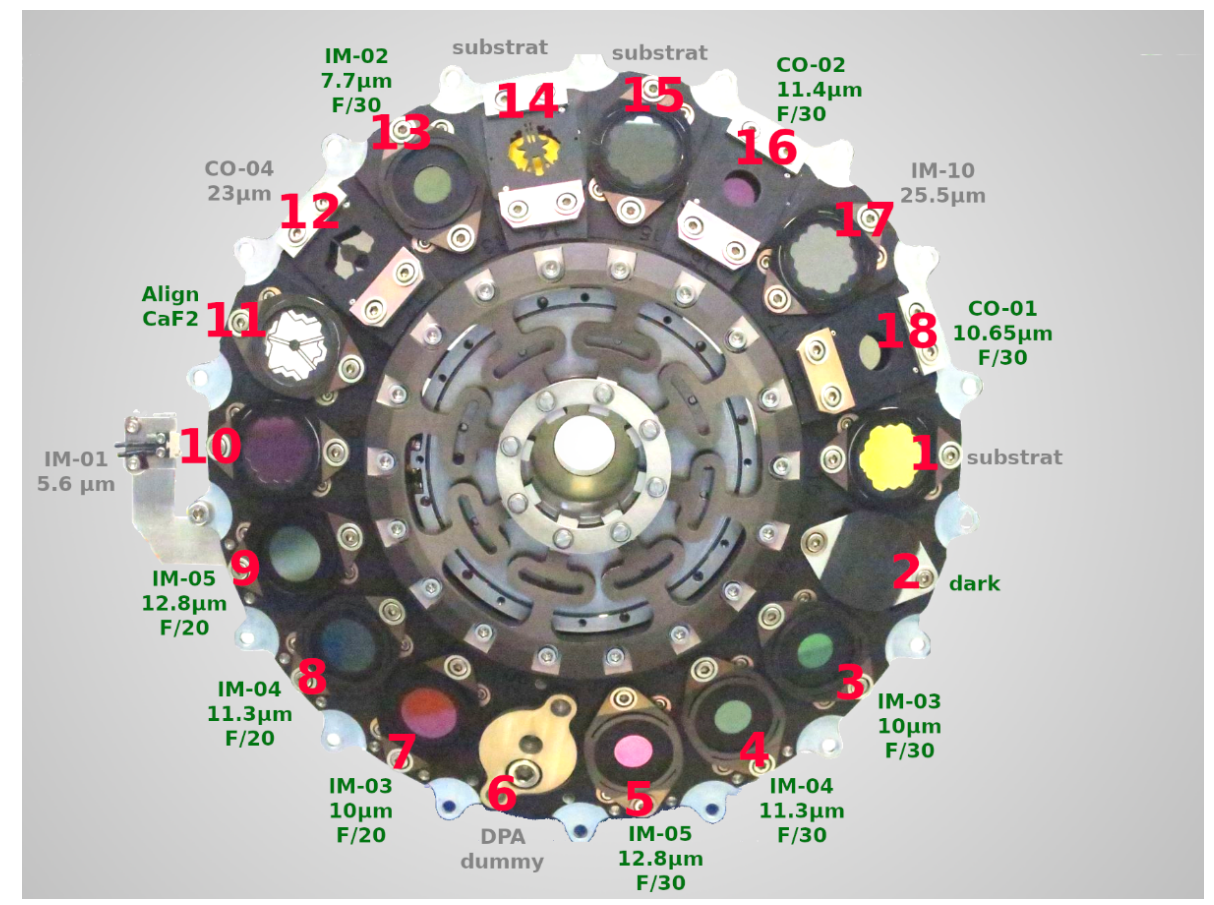

Figure 8. The filter wheel assembly equipped with MIRI filters and circular diaphragms suitable for the METIS AGPM testing. Some filters are duplicated, with a different diaphragm, allowing measurements with different beam F-ratio. 
Table 4. List of filters for the N-band coronagraphs tests.

\begin{tabular}{|c|c|c|}
\hline Filter & $\lambda_{0}(\mu \mathrm{m})$ & FWHM $(\mu \mathbf{m})$ \\
\hline IM-02 & 7.7 & 2.2 \\
\hline IM-03 & 10.00 & 2.0 \\
\hline IM-04 & 11.3 & 2.2 \\
\hline IM-05 & 12.8 & 2.4 \\
\hline CO-01 & 10.65 & 0.5 \\
\hline CO-02 & 11.4 & 0.6 \\
\hline Alignment lens & - & - \\
\hline Closed & - & - \\
\hline
\end{tabular}

equipped with diaphragms of various diameter, when possible (i.e. when the corresponding filter is duplicated). See Fig. 8.

\subsection{The Aquarius detector}

\subsubsection{Integration in CEA test bench}

The Aquarius detector is a standard mature deliverable, already supplied by ESO to two VLT projects. The system was initially developed for the upgrade of the VISIR instrument, in which two new Aquarius detector systems have been installed. These have been in operation, reliably at the telescope, for six years. Likewise an identical system was delivered to the MATISSE project, which is now also running at the telescope.

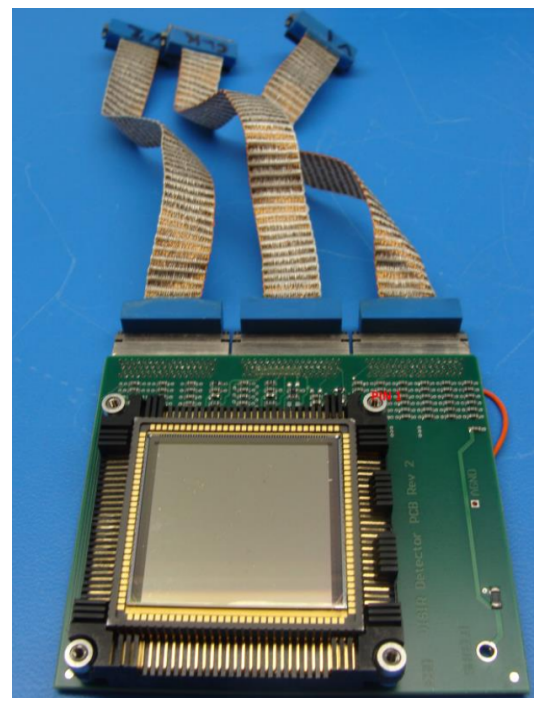

Figure 9. Aquarius detector on its PCB support frame, with cryogenic cables.

For the purpose of the CEA N-band test bench, the detector system breaks down into:

- the detector,

- the focal plane mount,

- the cryogenic cables,

- the preamplifier (at room temperature),

- the warm cables,

- the warm electronics (NGC), 
- the data acquisition computer.

In the CEA N-band test bench, the Aquarius runs at $8.5 \mathrm{~K}$ (best operating range is $7 \mathrm{~K}-9 \mathrm{~K}$ ). The thermal sensitivity (the dark current drift of the detector output signal with temperature) of the Aquarius detector is about $50-200 e^{-} / K$ around the operating temperature. The DC drift of the reference pixels could be slightly different compared to the active detector pixels and hence reference pixel subtraction may not entirely compensate for the thermal drifts during an exposure. Hence the operating temperature of the detector should be stabilized by active thermal controls using an ESO standard Lakeshore controller. To ensure the thermal drift contribution to the best noise achievable is negligible, the detector mount should be thermally stabilised to better than $10 \mathrm{mK}$ over typical exposure times foreseen during the tests. This is achieved with the standard setup used for the detector mount, with the usual DT-670 temperature diode, heater resistor and Lakeshore controller.

\subsubsection{Detector Readout architecture}

The architecture of the detector is such that it is split into two areas each of 512 rows and 1024 columns, at the top and bottom of the device, as shown in Fig. 10. Each area has 32 outputs, such that each output is configured to read out $32 \times 512$ pixels, all 64 outputs from the two areas being read in parallel. This readout scheme also allows for 16 outputs rather than 64 to simplify system design for low background applications. With this multiplexer configuration it is possible to read the detector out at $150 \mathrm{~Hz}$ frame rates, each output operational at $3 \mathrm{MHz}$ pixel rates. A windowed readout is also possible, where a user defined number of rows can be read, from the center outwards, with the remaining rows reset automatically. For example, a 1024 x 150 sized window, centred in the middle of the device, can be readout at $1 \mathrm{kHz}$ frame rates. There is no advantage to perform windowing in the column direction since all outputs run in parallel. All the typical detector read out modes are available and user-programmable with the simple control of five external clock control lines. These readout modes include:

- Global Reset where all pixels are reset at the same time

- Rolling mode, where a row is read then immediately reset, then the next row is read and reset and so on, for a programmable row time which can overlap the read of the next row;

- Non-Destructive Read without reset and Row Reset Level Read (RRLR) where a row can be reset then read.

Correlated Double Sampling, Fowler Sampling and Up-The-Ramp Sampling can all be implemented using any combination of these different read out modes.

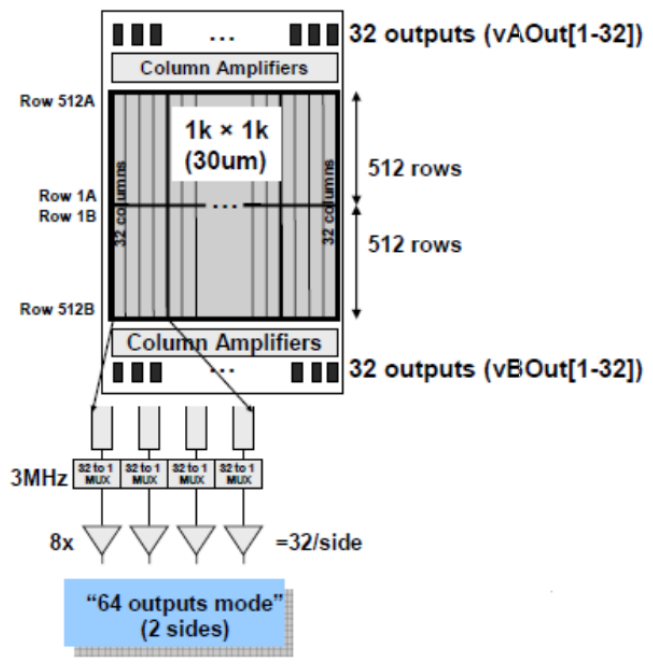

Figure 10. Detector architecture 
ESO has developed a standard cryogenic detector setup for a single Aquarius detector based application. It is a very compact design consisting of a detector mount and associated PCB which operates between $6 \mathrm{~K}$ and 9K. It also has a preamplifier (possibly set in the cryogenic environment), mounted a short distance away and which can operate at $50 \mathrm{~K}$ and above. It then connects to hermetic connectors and thence to the associated warm electronics and the NGC system. A standard Lakeshore temperature control system ensures the thermal stability that is required for Aquarius operations.

The NGC system gathers the detector controller rack split in three boards (two video boards 32 channels$10 \mathrm{MHz}$ and one front-end electronic board), the power supply rack, the temperature controller and the local control unit. All these devices are mounted in a single cabinet, as shown in Fig. 11.

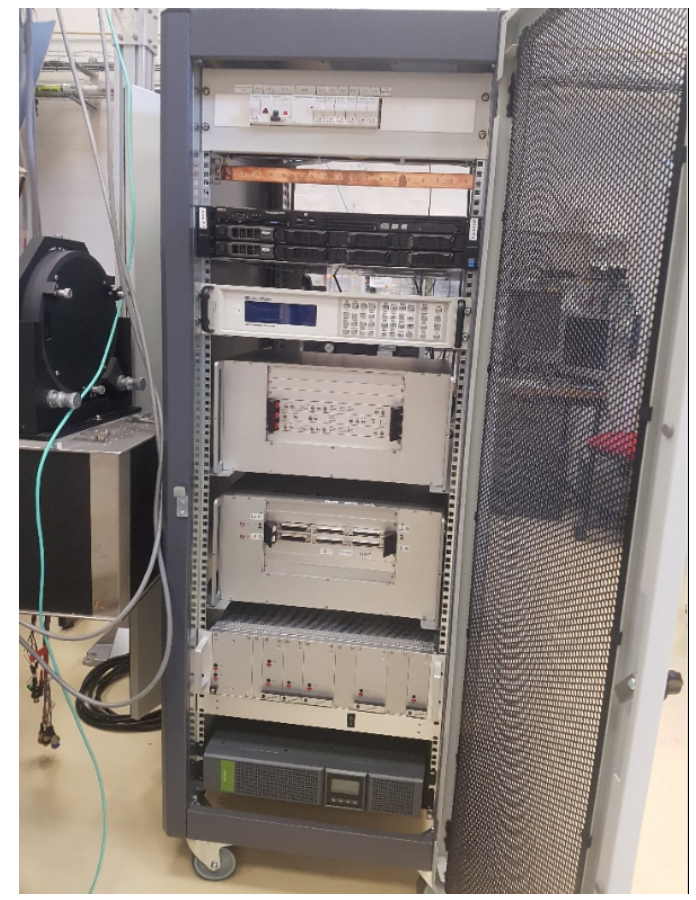

Figure 11. The NGC cabinet

\section{THE AGPMS}

The Annular Groove Phase Mask (AGPM) is a type of Vortex Phase Mask (VPM). A VPM is a transmissive optical component that creates a helical phase ramp around its optical axis. The phase ramp is described as $e^{i l \theta}$, where $\theta$ is the focal plane azimuthal coordinate and $l$ the (even) topological charge of the vortex. In the case of METIS, the baseline is to use charge-2 vortex phase masks (i.e., $l=2$ ). The VPMs of METIS will be based on the sub-wavelength grating technology, which has demonstrated appropriate performance for the METIS wavelength range (from 2.9 to $13.5 \mu \mathrm{m}$ ) in the lab and on 10-meter class telescopes. Sub-wavelength gratings have the property to create artificial birefringence (referred to as "form birefringence"). A concentric, circular grating can produce a space-variant half-wave plate when its parameters are properly tuned. This creates a vortex phase ramp with opposite signs for the two incoming circular polarizations. This concept for the VPM, referred to as the Annular Groove Phase Mask (AGPM), can be made sufficiently achromatic for the vortex phase mask to operate on a $30 \%$ bandwidth. Because of its limited bandwidth, a single VPM based on the AGPM concept cannot cover the whole METIS wavelength range in one shot. The METIS wavelength range will be divided as follows:

- VPM-L: 2.9 to $4.1 \mu \mathrm{m}$

- VPM-M: 4.0 to $5.3 \mu \mathrm{m}$ 
- VPM-N: 8.1 to $13.1 \mu \mathrm{m}$

Three N-band VPMs will eventually be installed side-by-side in a METIS focal plane to enable three-point chopping. These three N-band VPMs will be tested on the bench described in this paper. Each of them features different grating parameters (period, line width, and depth). The technology that will be used to produce the VPMs for METIS is based on reactive ion etching of an annular grating pattern onto a diamond substrate (aka the AGPM concept). The diamond substrate for the N-band AGPMs will be $15 \mathrm{~mm}$ in diameter and $300 \mu \mathrm{m}$ in thickness. An anti-reflective 2D square grating pattern will be etched on the back side to mitigate the effect of multiple internal reflections inside the substrate, and provide the highest possible transmission. SEM pictures of the front and back sides of the AGPM are displayed in Figure 12, and a photograph of an AGPM is shown in Figure 13.
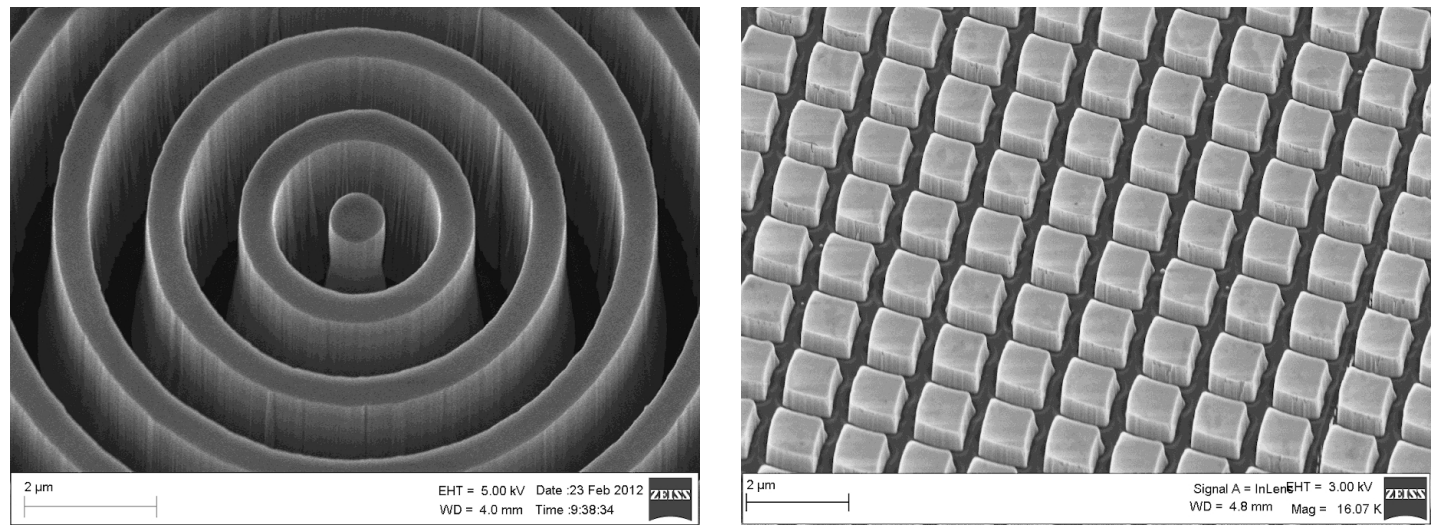

Figure 12. Left. SEM picture of the front side of an AGPM. Right. SEM picture of the back side of an AGPM.

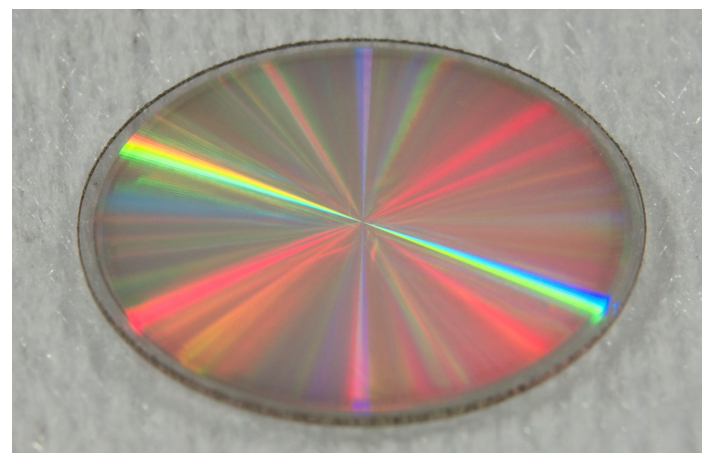

Figure 13. Picture of a VPM based on the AGPM concept.

\section{FIRST RESULTS}

The first test campaigns during the year 2020 were dedicated to various functional checks and learning how to use efficiently the set-up. For these tests, we used an N-band AGPM that had been manufactured for the VISIR instrument on VLT. This mask does not have the required performances, and we only use it to validate our measurement method.

\subsection{Comment about the setup}

There are two different setups with the fiber. First, on can use directly the output of the fiber as the input source of the telescope simulator. This approach makes sense, because this output, being single mode, is spatially coherent, and the source acts as a perfectly punctual one, which is how the AGPM performs the best. The beam 
has a Gaussian shape that is partially clipped by the telescope simulator pupil, and the PSF is something between a Gaussian and an Airy pattern. However, it appears that the beam is also elliptical (see Fig.2), which leads to an elliptical shape of the PSF.

The second setup is the one previously described (Sec. 2), with a pinhole on which the laser light is focused. The single-mode characteristic of the beam is partially lost, but a $10 \mu \mathrm{m}$ pinhole is sufficiently small so the coronagraph performances are not degraded, and the lasers are sufficiently powerful, so a good signal-to-noise ratio can be reached. The strong diffraction occurring at the pinhole breaks down the shape of the beam that exits the fiber, and the PSF recovers a round shape. The advantage of this set-up is also the possibility to have an efficient background subtraction, with a shutter mounted upstream of the pinhole.

Which set-up is best is still under discussion, and more tests with the fiber output directly may be performed in the future.

\subsection{Focus}

With an $\mathrm{F} / 30$ at $\lambda=10.5 \mu \mathrm{m}$, one is not very sensitive to focus, at least on a "normal" PSF (outside coronagraphic mask). However, with the coronagraphic mask, the effect of focus is well visible, and our first task was to finely adjust the focus. Figure 14 below shows the PSF under mask for various focus position. At best focus, the PSF is rotationally symmetrical as expected. We believe the residual astigmatism in the telescope simulator causes the funny PSF shape when defocus is applied
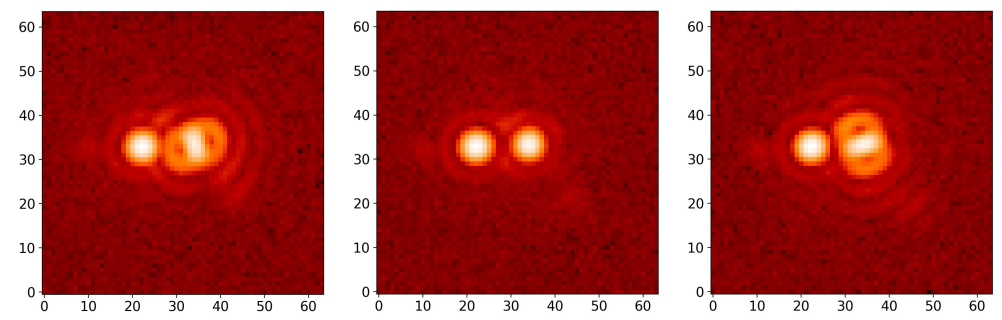

Figure 14. Coronagraphic PSFs with -5 (left), 0 (center) and +5 (right) mm of defocus, at $\lambda=10.5 \mu m$. The large spot to the left of the PSF is an expected and well known ghost image provoked by internal reflection in the cold neutral density

\subsection{Measurement method}

We followed the method as described below.

- For each laser, eight images around the mask centre are taken, on a regular grid, at a distance of $3 \mathrm{~mm}$ from the mask. Three millimetre is 38 pixels and 9.5x FWHM at $\lambda=10.5 \mu \mathrm{m}$.

- The source is then driven to the centre of the mask.

- From here, the source position is optimized, by moving it with manual precision translation stages.

- Image integration begins, with a DIT (detector integration time) usually 10 times larger than for the reference images.

- For each image, the signal is the encircled energy in a circle of diameter 1x FWHM. The reference signal is the average of the eight signals measured in each reference image, and the coronagraphic signal is measured in the image on the mask. The rejection factor is the ratio of the reference signal and the coronagraphic signal.

\subsection{Results}

\subsubsection{Rejection rate}

Table 5 gives the rejection rates obtained with this method. The values are below what was expected (the AGPM we tested was expected to give a rejection rate of about 150), especially at the short and long wavelength. Furthemore, the measured flux in the reference PSFs appears to vary significantly within the field. This is currently under investigation. See Sec. 5. 


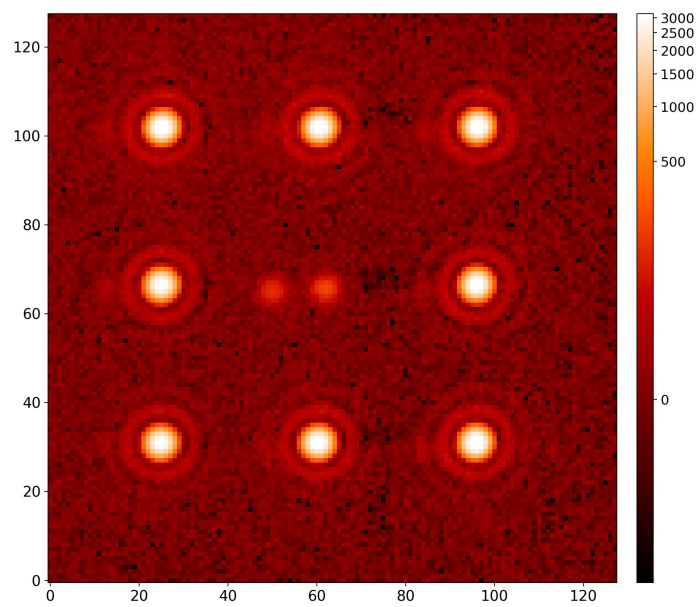

Figure 15. Illustration of the measurement method: 8 images at a distance of $10 x F W H M$ around the center of the mask +1 image on the mask. When on the mask, the ghost due to internal reflection in the cold neutral density is of similar intensity than the coronagraphic PSF

Table 5. Rejection factors measured at the 5 wavelengths provided by the QCLs. The accuracy given here is only based on the dispersion of the energy measured in the reference images.

\begin{tabular}{|c|c|c|c|c|c|}
\hline Wavelength & $8.0 \mu \mathrm{m}$ & $9.0 \mu \mathrm{m}$ & $10.5 \mu \mathrm{m}$ & $11.5 \mu \mathrm{m}$ & $12.5 \mu \mathrm{m}$ \\
\hline Rejection factor & $14 \pm 3$ & $77 \pm 12$ & $110 \pm 17$ & $99 \pm 6$ & $36 \pm 6$ \\
\hline
\end{tabular}

\subsubsection{PSFs profile}

Figure 16 shows the PSF profiles measured on and outside the mask at 9.0, 10.5 and $12.5 \mu \mathrm{m}$. The expected coronagraphic PSF is a scaled down version of the original PSF.
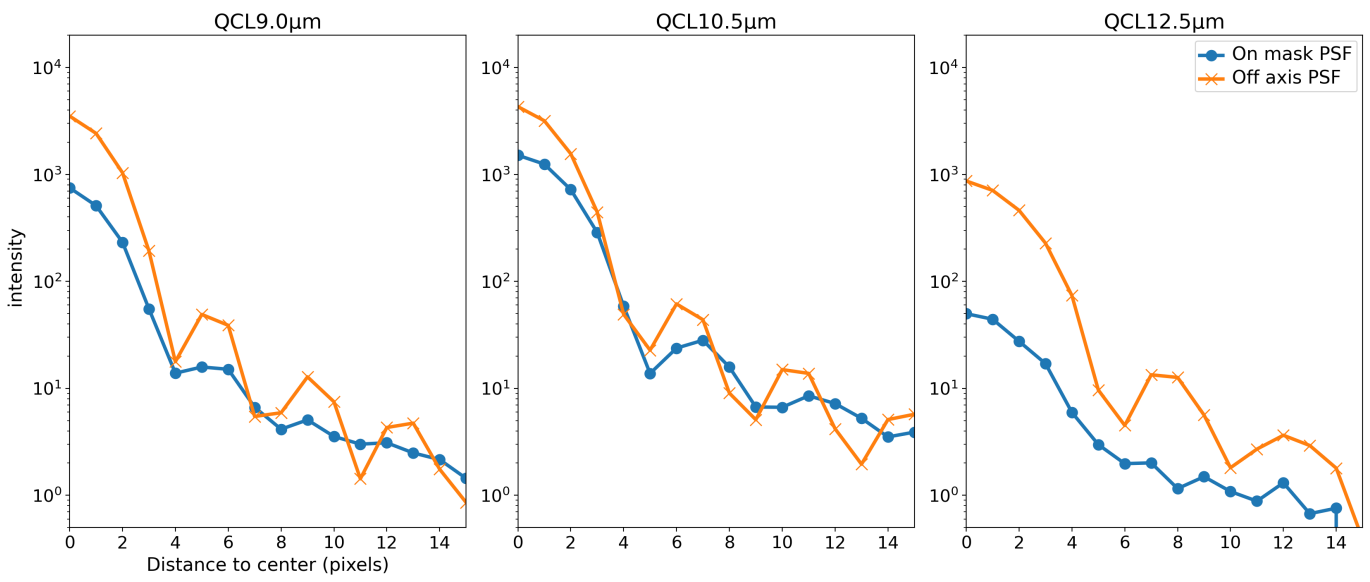

Figure 16. Profiles of the PSF showing the effect of the AGPM at different wavelengths. The "on-mask" PSF (blue dots) is a scaled down version of the "off-mask" PSF (orange crosses)

\section{CONCLUSION}

The test bench has the required performances to test the METIS AGPMs: the source can be finely moved on the mask, the signal-to-noise ratio is high, the stability is satisfactory. 
A test AGPM has been successfully mounted on the bench and measured. This AGPM is an "old" one (built for another project) and is known to have lower performances than the "real" METIS AGPMs.

However, the rejection rate measured are not at the level expected, by a factor x1.5 in the best case. An improvement could come from a better alignment of the telescope simulator, to remove residual astigmatism. Furthermore, the measurement accuracy is strongly impacted by the lack of a good reference PSF. The strong variation of the photometry within the field are currently under investigation. A first improvement came with a flat field correction, but it appears to be not sufficient. Figure 17 shows the photometry on a PSF at $12.5 \mu \mathrm{m}$ that has been moved around the mask. It shows that the variations in the PSF photometry are not dominated by the flat field. The laser light, with its long coherence length, can be subjected to many interferences (with the various coating, the diamonds substrate), which could explain the photometric variations.

Genuine METIS AGPM are planned to be tested early 2022.
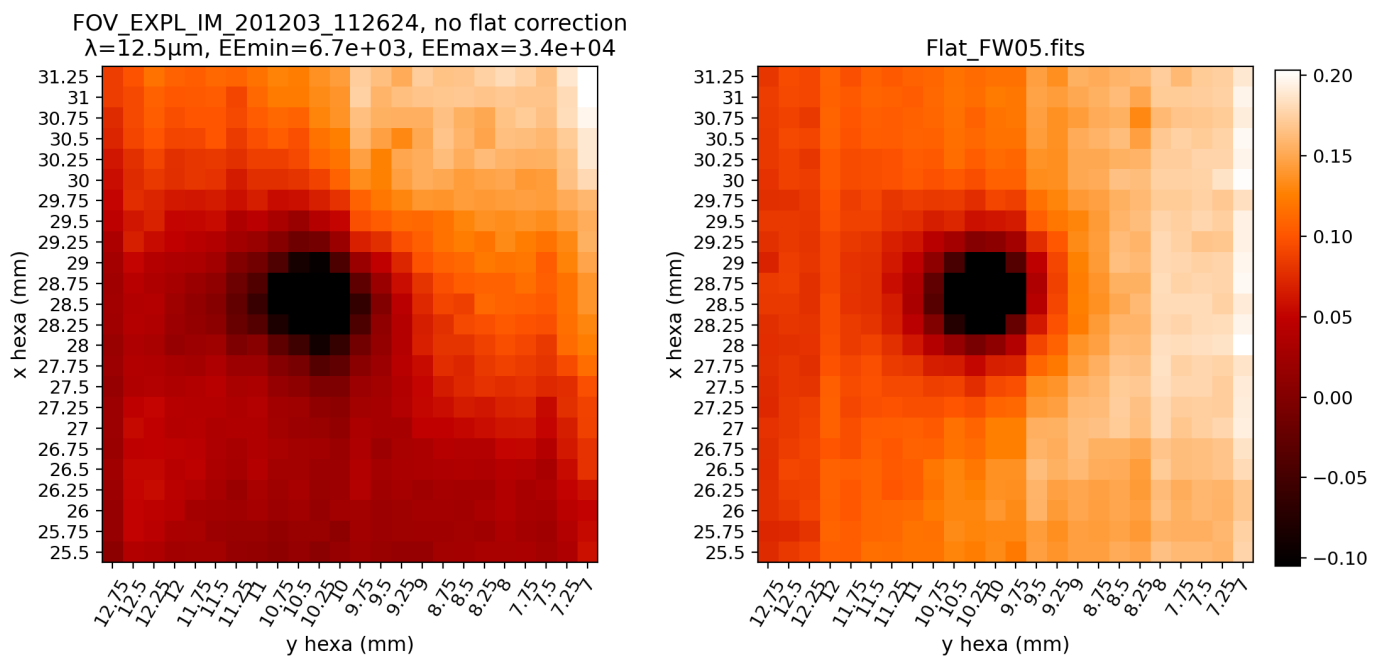

Figure 17. Left. Photometry on a PSF at $12.5 \mu \mathrm{m}$ moved around the center of the AGPM. The PSF is moved by steps of $250 \mu \mathrm{m}$ on a $24 \mathrm{x} 24 \mathrm{grid}$, hence covering a $6 x 6 \mathrm{~mm}^{2}$ area. The colors scale is the energy measured in the PSF relatively to the median value. Variation between $-10 \%$ and $+20 \%$ are observed (central part discarded). Right. The flat field of the corresponding area. It cannot explain fully the photometry variations observed. The center of the AGPM appears clearly on these images.

\section{ACKNOWLEDGMENTS}

Part of this work has received funding from the European Research Council (ERC) under the European Union's Horizon 2020 research and innovation programme (grant agreement No 819155). 